\title{
HIGHER-DERIVATIVE BOSON FIELD THEORIES AND CONSTRAINED SECOND-ORDER THEORIES
}

\author{
by \\ F.J. de $\operatorname{Urries}^{(*)(\dagger)}$, J.Julve ${ }^{(\dagger)}$ and E.J.Sánchez $(\dagger)(\diamond)$ \\ ${ }^{*}$ Departamento de Física, Universidad de Alcalá de Henares, \\ 28871 Alcalá de Henares (Madrid), Spain \\ (†) IMAFF, Consejo Superior de Investigaciones Científicas, \\ Serrano 113 bis, Madrid 28006, Spain \\ (») Departamento de Matemática, Universidad Europea, \\ 28670 Villaviciosa de Odón (Madrid), Spain
}

\begin{abstract}
As an alternative to the covariant Ostrogradski method, we show that higherderivative relativistic Lagrangian field theories can be reduced to second differentialorder by writing them directly as covariant two-derivative theories involving Lagrange multipliers and new fields. Despite the intrinsic non-covariance of the Dirac's procedure used to deal with the constraints, the explicit Lorentz invariance is recovered at the end. We develop this new setting on the grounds of a simple scalar model and then its applications to generalized electrodynamics and higher-derivative gravity are worked out. For a wide class of field theories this method is better suited than Ostrogradski's for a generalization to $2 n$-derivative theories.
\end{abstract}

PACS numbers: 11.10.Ef, 11.10.Lm, 04.60

e-mail addresses: julve@imaff.cfmac.csic.es; fernando.urries@alcala.es;

ejesus.sanchez@mat.ind.uem.es 


\section{Introduction}

Higher-derivative (HD) theories have an old tradition in physics. Relativistic field theories with higher order Lagrangians historically range from Higgs model regularizations [1] to generalized electrodynamics [2][3] and HD gravity [4], and arise as effective low energy theories of the string [5] or induced by quantum fields in a curved background [6]. A procedure was later devised to reduce them, by a Legendre transformation, to equivalent lower-derivative (LD) second-order theories [7] where a subsequent diagonalization explicitly displays the particle degrees of freedom [3][8][9].

The validity of the formal Lorentz covariant order-reducing method adopted there has been checked in an example of scalar HD theories by a rigorous study of the phase-space [9]. In this procedure, a generalization of the Ostrogradski formalism to continuous relativistic bosonic systems ( $2 n$-derivative because of Lorentz invariance in the most usual cases) is carried out. In it, some of the field derivatives and the generalized conjugate momenta become, after a suitable diagonalization, new field coordinates describing the degrees of freedom (DOF) which were already identified in the particle propagators arising in the algebraic decomposition of the HD propagator.

By using Lagrange multipliers, an alternative to the Ostrogradski method for mechanical discrete systems has been proposed which allows to show the quantum (Path Integral) equivalence between the modified action principle (first order Helmholtz Lagrangian) and the starting HD theory [10]. For relativistic field systems, we show that a similarly inspired procedure can be followed in which the multipliers let to write the HD theory from the outset as a second order (constrained) covariant one which lends itself to a particle interpretation after diagonalization.

In this paper we implement this new setting by means of the use of Lagrange multipliers in a Lorentz invariant formulation of relativistic scalar (and subsequent applications to vector and tensor) field theories. In the following we will generically refer to them as HD theories (HDT). The Dirac method [11] prescribes the identification of the primary constraints arising in the definition of the momenta. These constraints are added to the starting Hamiltonian by means of new multipliers, and then they are required to be conserved by the time evolution driven by this enlarged "total Hamiltonian" through the Poisson Bracket (PB). This may give rise to secondary constraints, the conservation of which can in turn generate more secondary constraints. The process stops when the equation obtained is not a constraint but an equation allowing the determination of a multiplier. We then use the remaining constraints to eliminate the remaining multipliers and the momenta, ending up with a two-derivative theory depending on just its true DOF. Since the latter appear mixed, a diagonalization works finally out the independently propagating DOF.

As long as time evolution is analyzed, the true mechanical Hamiltonian (i.e. the energy) of the system must be used. Then one cannot benefit of the compactness of the Lorentz invariant procedures introduced in [9], so we are initially forced to deal with non covariant $3+1$ objects and face the diagonalization of larger matrices. The 
relativistic invariance of the system becomes explicit only at the end of the process. Alternatively this invariance may also be maintained at an earlier stage by merging the constraints into covariant equations: in fact it is possible to find all the previously known 2nd class (non-covariant) Dirac's constraints by working out the whole set of covariant field equations (for fields and multipliers).

From the methodological point of view, the new treatment of HD boson theories that we present here provides a sharp departure from the more traditional Ostrogradski approach. Moreover, it is implementable and may prove advantageous in generalized electrodynamics, HD Yang-Mills and linearized HD gravity as well. On the other hand it lends itself to generalization to arbitrary $n$ better than the Ostrogradski method does.

Our work focuses on the propagating DOF of these theories. Therefore we mainly consider the free part of the corresponding (HD and their equivalent LD) Lagrangians. Self interactions (derivative or not) and interactions with other (external) fields are embodied in a source term, namely a source $j$ linearly coupled to the fundamental field. The source always contains a coupling constant. Thus for our purposes one may adopt two attitudes: either $j$ is assumed to contain only (spectator) external fields or the whole source term is treated as a perturbation (and ignored at the zeroth order in the coupling we are interested in) if self couplings are present. In any case we retain an external source as a guide to find the suitable diagonalizing matrix.

HD (free) fermion theories will always be $(2 n+1)$-derivative to maintain Lorentz invariance. The constraint method presented here (as well as Ostrogradski's) can be adapted to encompass these theories, but the task is beyond the purposes of this paper.

Again resorting to the scalar example, in Section 2 we review the general situation of HD theories in which the "square masses" may be the complex and/or degenerate roots of a polynomial, then justifying the custom of focusing only on theories with real non-degenerate squared masses. In Section 3 we treat $n=3$, the case $n=2$ being too much trivial for our illustrative purposes. The results regarding the extension to arbitrary $n$ are presented in Section 4. Through the Dirac's canonical method followed, they guarantee that all the constraints present in the theory have been properly taken into account. This validates the Lorentz covariant phase-space method described in Section 5 which we adopt in the following as the suitable shortcut for practical uses. Section 6 discusses, with the appropriate mathematical formalism, the application of our constraint method to HD (Podolsky) vector field theories and generalizations together the most interesting case of HD (linearized) gravity. We summarize our results in the Conclusions in Section 7.

In Appendix A we show the pure algebraic character (i.e. absence of space derivatives) of the secondary constraints. Also for the general $2 n$-derivative case, in Appendices $\mathrm{B}$ and $\mathrm{C}$ we display the steps leading to the diagonalization of the DOF. In Appendix D, when squares of the multipliers occur, we show the equivalence between this seemingly constraint method and Ostrogradki's.

Throughout the paper the Minkowski metric (+- - ) is adopted. 


\section{HD Scalar Theories}

The theories of arbitrary (finite) differential order have a distinctive spectrum with respect to the usual second order theories describing physical systems. It generally includes peculiar ghostly states and non-particle solutions. Although this feature is not exclusive of HDT's (one may devise second order theories with this sort of complications), it is almost inherent to them. The exception corresponds to very special cases like HD gravity made up only with powers of the scalar curvature. There is no escape however for simpler fields.

The simplest HDT's are linear theories of scalar fields

$$
\mathcal{L}^{N}=-\frac{c_{N}}{2} \phi q_{N}(\square) \phi-j \phi
$$

where $c_{N}$ is a constant with suitable dimensions and $q_{N}(\square)$ is a monic real polynomial in $\square$. A detailed study of the theories (2.1) in which $q_{N}$ has only real non-degenerate roots can be found in [9]. In these cases, the theory (2.1) is equivalent to a LD one, made up of the alternate sum of $N$ Klein-Gordon (KG) free Lagrangians where the sum of the respective real fields couples to the source $j$. This states that all the DOF are particle-like and couple to the same source.

However this is a privileged case of (2.1) as long as $q_{N}$ may generally have degenerate and/or complex roots

$$
\begin{aligned}
q_{N}(\square)= & \left(\square+m_{1}^{2}\right)^{R_{1}} \cdots\left(\square+m_{r}^{2}\right)^{R_{r}}\left(\square-M_{1}^{2}\right)^{T_{1}} \cdots\left(\square-M_{1}^{2}\right)^{T_{t}} \\
& {\left[\left(\square-\Omega_{1}\right)\left(\square-\bar{\Omega}_{1}\right)\right]^{C_{1}} \cdots\left[\left(\square-\Omega_{c}\right)\left(\square-\bar{\Omega}_{c}\right)\right]^{C_{c}} }
\end{aligned}
$$

where the $m_{i}$ correspond to physical masses, $M_{i}$ are tachionic and $\Omega_{i}$ are complex, $R_{i}, T_{i}$ and $C_{i}$ being their respective degeneracies.

Non degenerate complex roots can be handled formally with the methods developed in [9]. Following them, it can be seen that

$$
\mathcal{L}^{4}=-\frac{c_{4}}{2} \phi(\square-\Omega)(\square-\bar{\Omega}) \phi-j \phi
$$

is equivalent to

$$
\mathcal{L}^{2}=\frac{c_{4}}{2}(\Omega-\bar{\Omega})[\varphi(\square-\Omega) \varphi-\bar{\varphi}(\square-\bar{\Omega}) \bar{\varphi}]-j(\varphi+\bar{\varphi}),
$$

where $\varphi=\varphi_{1}+i \varphi_{2}$ is a complex scalar field. However this is not a real diagonalization because (2.2) cannot be diagonalised in terms of real (or even complex) scalar fields built linearly out of $\varphi_{1}$ and $\varphi_{2}$ and with real square masses.

Also the degeneracy makes it impossible to interpret the roots as the square masses of free particle-like (even tachionic) states. In fact, already the simple case $\phi\left(\square+m^{2}\right)^{2} \phi$ cannot be reduced to a LD theory of free real (or complex) fields because the HD propagator $1 /\left(\square+m^{2}\right)^{2}$ is not a linear combination of LD free propagators $1 /\left(\square \pm m_{i}^{2}\right)$. Otherwise stated, there are solutions to $\left(\square+m^{2}\right)^{2} \phi=0$ that are not solutions to $\left(\square+m^{2}\right) \phi=0$, and hence cannot be expressed as superpositions of plane waves.

Summarizing, only in the case of real non-degenerate square masses can the HD propagator be algebraically expanded as a sum of particle-like propagators, i.e. in terms of propagators displaying physical or tachionic masses (sign of the square mass), or even a non-physical norm (sign of the propagator). As customary in the literature, in the following we will limit ourselves to the study of these cases. 


\section{3. $\mathbf{n}=3$ theory}

We consider the six-derivative Lagrangian,

$$
\mathcal{L}^{6}=-\frac{1}{2} \frac{\mu^{2}}{M} \phi \llbracket 1 \rrbracket \llbracket 2 \rrbracket \llbracket 3 \rrbracket \phi-j \phi
$$

where $\mu$ is an arbitrary mass parameter, $\llbracket i \rrbracket \equiv\left(\square+m_{i}^{2}\right)$ are KG operators, $M \equiv$ $\langle 12\rangle\langle 13\rangle\langle 23\rangle,\langle i j\rangle \equiv m_{i}^{2}-m_{j}^{2}>0$ for $i<j$, and mass dimensions $[\mu]=1,[M]=6$, $[\phi]=1,[j]=3$. We have overlooked the case $n=2$ because it is too trivial for introductory purposes.

As discussed in [9], (3.1) displays the general form of the free part of a higherderivative scalar theory with non-degenerate masses $m_{1}, m_{2}, m_{3}$, the source term embodying the remaining self-interactions and the couplings to other fields. There we showed by a covariant Legendre order-reduction procedure that (3.1) is equivalent to the second-order Lagrangian

$$
\mathcal{L}^{2}=-\frac{1}{2} \frac{\mu^{2}}{\langle 23\rangle} \phi_{1} \llbracket 1 \rrbracket \phi_{1}+\frac{1}{2} \frac{\mu^{2}}{\langle 13\rangle} \phi_{2} \llbracket 2 \rrbracket \phi_{2}-\frac{1}{2} \frac{\mu^{2}}{\langle 12\rangle} \phi_{3} \llbracket 3 \rrbracket \phi_{3}-j\left(\phi_{1}+\phi_{2}+\phi_{3}\right)
$$

in agreement with what is expected from the algebraic expansion of the HD propagator in particle poles, namely

$$
-\frac{\mu^{-2} M}{\llbracket 1 \rrbracket \llbracket 2 \rrbracket \llbracket 3 \rrbracket}=-\frac{\mu^{-2}\langle 23\rangle}{\llbracket 1 \rrbracket}+\frac{\mu^{-2}\langle 13\rangle}{\llbracket 2 \rrbracket}-\frac{\mu^{-2}\langle 12\rangle}{\llbracket 3 \rrbracket} .
$$

The physical meaning is that whenever a source $j$ emits a field $\phi$ driven by a HD dynamics, it actually emits a linear superposition $\phi=\phi_{1}+\phi_{2}+\phi_{3}$ of LD (particle) fields.

However $\mathcal{L}^{6}$ can be recast directly as a second-order theory with constraints, namely

$$
\mathcal{L}^{6}=\frac{1}{2} \frac{\mu^{2}}{M}\left[-\bar{\psi}_{3} \llbracket 1 \rrbracket \bar{\psi}_{1}+\lambda_{1}\left(\bar{\psi}_{1}-\llbracket 2 \rrbracket \bar{\psi}_{2}\right)+\lambda_{2}\left(\bar{\psi}_{2}-\llbracket 3 \rrbracket \bar{\psi}_{3}\right)\right]-j \bar{\psi}_{3} \quad,
$$

where $\bar{\psi}_{3}=\phi$ and $\lambda_{1}, \lambda_{2}$ are Lagrange multipliers, so that $\mathcal{L}^{6}$ depends on five fields. It is immediate to check that the equations of motion for $\lambda_{1}$ and $\lambda_{2}$ yield $\bar{\psi}_{1}$ and $\bar{\psi}_{2}$ as (derivative) functions of $\bar{\psi}_{3}$. By substituting them in (3.4) and identifying $\bar{\psi}_{3}$ and $\phi$ one recovers the HD equation (3.1). Dropping a total time-derivative, in compact matrix notation, (3.4) reads

$$
\mathcal{L}^{6}=\frac{1}{2} \dot{\Psi}^{T} \mathcal{K} \dot{\Psi}+\frac{1}{2} \Psi^{T} \mathcal{M} \Psi-J^{T} \Psi
$$


where the vectors $\Psi$ and $J$, with components $\psi_{i}, J_{i}$, and the matrices $\mathcal{K}$ and $\mathcal{M}$ are

$$
\begin{gathered}
\Psi \equiv\left(\begin{array}{c}
\mu^{-4} \bar{\psi}_{1} \\
\mu^{-2} \bar{\psi}_{2} \\
\bar{\psi}_{3} \\
\mu^{-2} \lambda_{1} \\
\mu^{-4} \lambda_{2}
\end{array}\right) \text { so that }\left[\psi_{i}\right]=1 \quad i=1, \ldots, 5 \quad ; \quad J_{i}=j \delta_{3 i} \quad ; \\
\mathcal{K}=\frac{\mu^{6}}{2 M}\left(\begin{array}{lllll}
0 & 0 & 1 & 0 & 0 \\
0 & 0 & 0 & 1 & 0 \\
1 & 0 & 0 & 0 & 1 \\
0 & 1 & 0 & 0 & 0 \\
0 & 0 & 1 & 0 & 0
\end{array}\right) ; \mathcal{M}=\frac{\mu^{6}}{2 M}\left(\begin{array}{ccccc}
0 & 0 & -M_{1}^{2} & \mu^{2} & 0 \\
0 & 0 & 0 & -M_{2}^{2} & \mu^{2} \\
-M_{1}^{2} & 0 & 0 & 0 & -M_{3}^{2} \\
\mu^{2} & -M_{2}^{2} & 0 & 0 & 0 \\
0 & \mu^{2} & -M_{3}^{2} & 0 & 0
\end{array}\right) .
\end{gathered}
$$

$\mathcal{M}$ is an operator with space derivatives present in $M_{i}^{2} \equiv m_{i}^{2}-\Delta$.

The canonical conjugate momenta are defined as

$$
\pi_{i}=\frac{\partial \mathcal{L}^{6}}{\partial \dot{\psi}_{i}}
$$

They are the components of a 5 -vector $\Pi$ for which one has

$$
\Pi=\mathcal{K} \dot{\Psi}
$$

Since $\mathcal{K}$ is not invertible, not all the velocities $\dot{\psi}_{i}$ can be expressed in terms of the momenta and a primary constraint occurs, namely

$$
\Omega_{1} \equiv \pi_{5}-\pi_{1}=0
$$

as consequence of $\pi_{5}=\frac{\mu^{6}}{2 M} \dot{\psi}_{3}=\pi_{1}$. There is only one such a constraint since the submatrix $\mathcal{K}_{a b} \equiv \frac{\mu^{6}}{2 M} \mathcal{K}_{a b}^{\prime} \quad(a, b=1, \ldots, 4)$ is regular. In the following, indices $a, b, \ldots$ go from 1 to 4 , while $i, j, \ldots$ go from 1 to 5 . The velocity $\dot{\psi}_{5}$ is not worked out, and from (3.8) we have

$$
\pi_{a}=\frac{\mu^{6}}{2 M} \mathcal{K}_{a b}^{\prime} \dot{\psi}_{b}+\frac{\mu^{6}}{2 M} \delta_{a 3} \dot{\psi}_{5}
$$

and therefore

$$
\dot{\psi}_{a}=\frac{2 M}{\mu^{6}} \mathcal{K}_{a b}^{\prime} \pi_{b}-\delta_{a 1} \dot{\psi}_{5}
$$


The Hamiltonian is

$$
\mathcal{H}=\pi_{a} \dot{\psi}_{a}+\pi_{5} \dot{\psi}_{5}-\frac{1}{2} \dot{\psi}_{a} \mathcal{K}_{a b} \dot{\psi}_{b}-\frac{\mu^{6}}{2 M} \dot{\psi}_{3} \dot{\psi}_{5}-\frac{1}{2} \psi_{i} \mathcal{M}_{i j} \psi_{j}+j \psi_{3}
$$

where $\dot{\psi}_{a}$ must be substituted according to $(3.11)$. Then the dependence on $\dot{\psi}_{5}$ cancels out and we have

$$
\mathcal{H}=\frac{1}{2} \frac{2 M}{\mu^{6}} \pi_{a} \mathcal{K}_{a b}^{\prime} \pi_{b}-\frac{1}{2} \psi_{i} \mathcal{M}_{i j} \psi_{j}+J_{i} \psi_{i}
$$

Since not all of the five momenta $\pi_{i}$ are independent because of the primary constraint (3.9), only four momenta appear in (3.13) together with the five fields $\psi_{i}$. The "total Hamiltonian", with five independent momenta, accounting for this is

$$
\mathcal{H}_{T}=\mathcal{H}+\zeta \Omega_{1}
$$

where $\zeta$ is a Lagrange multiplier.

The stability of $\Omega_{1}$ requires

$$
\dot{\Omega}_{1}=\left\{\Omega_{1}, \mathcal{H}_{T}\right\}_{P B} \equiv \Omega_{2}=\frac{\mu^{6}}{2 M}\left(\langle 13\rangle \psi_{3}-\mu^{2} \psi_{4}+\mu^{2} \psi_{2}\right)=0
$$

This secondary constraint yields

$$
\psi_{4}=\frac{\langle 13\rangle}{\mu^{2}} \psi_{3}+\psi_{2}
$$

Further secondary constraints stem from the ensuing stability conditions

$$
\dot{\Omega}_{2}=\left\{\Omega_{2}, \mathcal{H}_{T}\right\}_{P B} \equiv \Omega_{3}=\langle 13\rangle \pi_{1}-\mu^{2} \pi_{2}+\mu^{2} \pi_{4}=0
$$

so that

$$
\pi_{4}=\pi_{2}-\frac{\langle 13\rangle}{\mu^{2}} \pi_{1}
$$

and again

$$
\begin{aligned}
\dot{\Omega}_{3} & =\left\{\Omega_{3}, \mathcal{H}_{T}\right\}_{P B} \equiv \Omega_{4}= \\
& =\frac{\mu^{6}}{2 M}\left(-\langle 13\rangle\langle 23\rangle \psi_{3}-\mu^{2}\langle 13\rangle \psi_{2}-\mu^{4} \psi_{1}+\mu^{4} \psi_{5}\right)=0
\end{aligned}
$$


(once (3.16) has been used), from which one gets

$$
\psi_{5}=\frac{\langle 13\rangle\langle 23\rangle}{\mu^{4}} \psi_{3}+\frac{\langle 13\rangle}{\mu^{2}} \psi_{2}+\psi_{1}
$$

The next constraint, after using (3.18), gives

$$
\dot{\Omega}_{4}=\left\{\Omega_{4}, \mathcal{H}_{T}\right\}_{P B} \equiv \Omega_{5}=\langle 13\rangle\langle 12\rangle \pi_{1}-\mu^{2}\langle 13\rangle \pi_{2}-\mu^{4} \pi_{3}+2 \frac{\mu^{6}}{2 M} \mu^{4} \zeta=0 \quad,
$$

and $\zeta$ can be obtained as a function of $\pi_{1}, \pi_{2}$, and $\pi_{3}$, thus bringing the generation of secondary constraints to and end.

$\mathcal{H}_{T}$ being quadratic in $\psi$ 's and $\pi$ 's, guarantees an alternance of linear constraints involving the fields and the momenta. In spite of the occurrence of space derivatives in $\mathcal{M}$, they cancel out and the constraints are algebraic. From this set of constraints, the multipliers $\psi_{4}$ and $\psi_{5}$, together with their conjugate momenta $\pi_{4}$ and $\pi_{5}$, can be worked out.

The Lagrangian (3.5) can be expressed in terms of the independent variables $\psi_{\alpha} \quad(\alpha=1,2,3)$. Notice that implementing these algebraic constraints in $\mathcal{L}^{6}$ does not modify the second differential order already attained in (3.5). One obtains

$$
\mathcal{L}^{6}=\dot{\psi}_{\alpha} \overline{\mathcal{K}}_{\alpha \beta} \dot{\psi}_{\beta}+\psi_{\alpha} \overline{\mathcal{M}}_{\alpha \beta} \psi_{\beta}-j \psi_{3}
$$

where

$$
\begin{aligned}
& \overline{\mathcal{K}}_{\alpha \beta} \equiv \frac{1}{2}\left(\mathcal{K}_{\alpha \beta}+\mathcal{K}_{\alpha B} \mathcal{N}_{B \beta}+\mathcal{N}_{\alpha A} \mathcal{K}_{A \beta}\right)=\frac{\mu^{6}}{2 M}\left(\begin{array}{ccc}
0 & 0 & 1 \\
0 & 1 & \frac{\langle 13\rangle}{\mu^{2}} \\
1 & \frac{\langle 13\rangle}{\mu^{2}} & \frac{\langle 13\rangle\langle 23\rangle}{\mu^{4}}
\end{array}\right) \\
& \overline{\mathcal{M}}_{\alpha \beta} \equiv \frac{1}{2}\left(\mathcal{M}_{\alpha \beta}+\mathcal{M}_{\alpha B} \mathcal{N}_{B \beta}+\mathcal{N}_{\alpha A} \mathcal{M}_{A \beta}\right)= \\
&=\frac{\mu^{6}}{2 M}\left(\begin{array}{ccc}
0 & \mu^{2} & -M_{3}^{2} \\
\mu^{2} & \langle 13\rangle-M_{2}^{2} & -\frac{\langle 13\rangle}{\mu^{2}} M_{3}^{2} \\
-M_{3}^{2} & -\frac{\langle 13\rangle}{\mu^{2}} M_{3}^{2} & -\frac{\langle 13\rangle\langle 23\rangle}{\mu^{4}} M_{3}^{2}
\end{array}\right)
\end{aligned}
$$

with $\alpha, \beta, \ldots=1,2,3 ; A, B, \ldots=4,5 ;$ and

$$
\mathcal{N}_{A \beta} \equiv\left(\begin{array}{ccc}
0 & 1 & \frac{\langle 13\rangle}{\mu^{2}} \\
1 & \frac{\langle 13\rangle}{\mu^{2}} & \frac{\langle 13\rangle\langle 23\rangle}{\mu^{4}}
\end{array}\right)
$$


that allows to embody (3.16) and (3.20) in the closed form

$$
\psi_{A}=\mathcal{N}_{A \beta} \psi_{\beta}
$$

The symmetric matrices $\overline{\mathcal{K}}$ and $\overline{\mathcal{M}}$ can be simultaneously diagonalized by the regular transformation

$$
\psi_{\alpha}=\mathcal{R}_{\alpha \beta} \phi_{\beta}
$$

where

$$
\mathcal{R}_{\alpha \beta} \equiv\left(\begin{array}{ccc}
\frac{\langle 12\rangle\langle 13\rangle}{\mu^{4}} & 0 & 0 \\
-\frac{\langle 13\rangle}{\mu^{2}} & -\frac{\langle 23\rangle}{\mu^{2}} & 0 \\
1 & 1 & 1
\end{array}\right)
$$

The reason for this property will be made clear later on.

The 3rd. row of the non-orthogonal matrix $\mathcal{R}$ in (3.28), has been chosen so as to yield the source term in (3.2), in which the source couples to the sum of the LD effective fields. The remaining six elements are uniquely determined by requiring $\mathcal{R}$ to diagonalize $\overline{\mathcal{K}}$ and $\overline{\mathcal{M}}$.

The diagonalized matrices are

$$
\begin{gathered}
\mathcal{R}^{T} \overline{\mathcal{K}} \mathcal{R}=\frac{\mu^{6}}{2 M} \operatorname{diag}\left(\frac{\langle 12\rangle\langle 13\rangle}{\mu^{4}},-\frac{\langle 12\rangle\langle 23\rangle}{\mu^{4}}, \frac{\langle 13\rangle\langle 23\rangle}{\mu^{4}}\right), \\
\mathcal{R}^{T} \overline{\mathcal{M}} \mathcal{R}=\frac{\mu^{6}}{2 M} \operatorname{diag}\left(-M_{1}^{2} \frac{\langle 12\rangle\langle 13\rangle}{\mu^{4}}, M_{2}^{2} \frac{\langle 12\rangle\langle 23\rangle}{\mu^{4}},-M_{3}^{2} \frac{\langle 13\rangle\langle 23\rangle}{\mu^{4}}\right),
\end{gathered}
$$

so that (3.22) finally writes as $\mathcal{L}^{2}$ in $(3.2)$.

This shows again the Ostrogradski-based result [9] of the equivalence between the six-derivative theory (3.1) and the LD version (3.2) that reproduces the propagator structure (3.3). 


\section{Theories with arbitrary $\mathbf{n}$}

The general Lagrangian

$$
\mathcal{L}^{2 n}=-\frac{1}{2} \frac{\mu^{d}}{M} \phi \llbracket 1 \rrbracket \llbracket 2 \rrbracket \ldots \llbracket n \rrbracket \phi-j \phi \quad,
$$

where $M \equiv \prod_{i<j}\langle i j\rangle$, and $d=n(n-3)+2$ for dimensional convenience, can be dealt with along similar lines. The 2-derivative constrained recasting of (4.1) is

$$
\mathcal{L}^{2 n}=\frac{1}{2} \frac{\mu^{d}}{M}\left[-\bar{\psi}_{n} \llbracket 1 \rrbracket \bar{\psi}_{1}+\lambda_{1}\left(\bar{\psi}_{1}-\llbracket 2 \rrbracket \bar{\psi}_{2}\right)+\ldots+\lambda_{n-1}\left(\bar{\psi}_{n-1}-\llbracket n \rrbracket \bar{\psi}_{n}\right)\right]-j \bar{\psi}_{n} \quad,
$$

with $\bar{\psi}_{n} \equiv \phi$, and $\lambda_{1}, \ldots, \lambda_{n-1}$ being Lagrange's multipliers. In order to have a more compact notation we define

$$
\begin{array}{ll}
\psi_{\alpha}=\mu^{-2(n-\alpha)} \bar{\psi}_{\alpha} & ; \quad \alpha=1, \ldots, n \\
\psi_{A}=\mu^{-2 \alpha} \lambda_{\alpha} & ; \quad A=n+\alpha ; \alpha=1, \ldots, n-1
\end{array}
$$

so that $\left[\psi_{i}\right]=1 \quad(i=1, \ldots, 2 n-1)$. Then

$$
\mathcal{L}^{2 n}=\frac{1}{2} \dot{\Psi}^{T} \mathcal{K} \dot{\Psi}+\frac{1}{2} \Psi^{T} \mathcal{M} \Psi-J^{T} \Psi
$$

with $J_{i}=j \delta_{i n}$, and the $(2 n-1) \times(2 n-1)$ matrices $\mathcal{K}$ and $\mathcal{M}$ are given by

$$
\begin{aligned}
\mathcal{K}_{i j} & \equiv \sigma\left(\delta_{i, j-n+1}+\delta_{j, i-n+1}\right) \\
\mathcal{M}_{i j} & \equiv \sigma\left[-\left(M_{\underline{i}}^{2} \delta_{i, j-n+1}+M_{\underline{j}}^{2} \delta_{j, i-n+1}\right)+\mu^{2}\left(\delta_{i, j-n}+\delta_{j, i-n}\right)\right],
\end{aligned}
$$

with $\sigma \equiv \frac{\mu^{n(n-1)}}{2 M}$. This "mass" matrix contains again space derivatives. Here and in the following an underlined index means that Einstein summation convention does not apply. The canonical conjugate momenta are now

$$
\pi_{i}=\frac{\partial \mathcal{L}^{2 n}}{\partial \dot{\psi}_{i}}
$$

i.e., in closed notation,

$$
\Pi=\mathcal{K} \dot{\Psi}
$$

Defining the matrix $\mathcal{K}^{\prime}$

$$
\mathcal{K}_{a b}^{\prime}=\frac{1}{\sigma} \mathcal{K}_{a b} \quad(a, b=1, \ldots, 2 n-2) \quad,
$$

one sees that $\operatorname{det} \mathcal{K}^{\prime} \neq 0$, while $\operatorname{det} \mathcal{K}=0$. This means that we only have one primary constraint, namely

$$
\Omega_{1} \equiv \pi_{2 n-1}-\pi_{1}=0 \quad .
$$


Then $\dot{\psi}_{2 n-1}$ is not worked out, while $\dot{\psi}_{a} \quad(a=1, \ldots, 2 n-2)$ can be expressed in terms of $\pi_{a}$ and $\dot{\psi}_{2 n-1}$. The first $2 n-2$ components of eq.(4.7), namely

$$
\pi_{a}=\sigma \mathcal{K}_{a b}^{\prime} \dot{\psi}_{b}+\sigma \delta_{a n} \dot{\psi}_{2 n-1}
$$

give

$$
\dot{\psi}_{a}=\frac{1}{\sigma} \mathcal{K}_{a b}^{\prime} \pi_{b}-\delta_{a 1} \dot{\psi}_{2 n-1}
$$

After checking that the terms in $\dot{\psi}_{2 n-1}$ cancel out, the Hamiltonian has the simple expression

$$
\mathcal{H}=\frac{1}{2} \sigma \pi_{a} \mathcal{K}_{a b}^{\prime} \pi_{b}-\frac{1}{2} \psi_{i} \mathcal{M}_{i j} \psi_{j}+j \psi_{n}
$$

In $\mathcal{H}$ only $2 n-2$ momenta $\pi_{a}$ occur against $2 n-1$ fields $\psi_{i}$, because of the primary constraint (4.9). One may restore the dependence on $2 n-1$ momenta by introducing the "total Hamiltonian"

$$
\mathcal{H}_{T}=\mathcal{H}+\zeta \Omega_{1}
$$

where $\zeta$ is a Lagrange multiplier.

From the stability condition on $\Omega_{1}$, a cascade of secondary constraints follows, eventually ending with an equation that determines the value of $\zeta$. We outline here the steps closely following the lines of section 3 .

$$
\dot{\Omega}_{1}=\left\{\Omega_{1}, \mathcal{H}_{T}\right\}_{P B} \equiv \Omega_{2}=0 \quad \Rightarrow \quad \psi_{n+1}=\psi_{n-1}+\frac{\langle 1 n\rangle}{\mu^{2}} \psi_{n}
$$

Then

$$
\dot{\Omega}_{2}=\left\{\Omega_{2}, \mathcal{H}_{T}\right\}_{P B} \equiv \Omega_{3}=\mu^{2} \pi_{2 n-2}+\langle 1 n\rangle \pi_{1}-\mu^{2} \pi_{2}\left(1-\delta_{n 2}\right)-2 \sigma \zeta \delta_{n 2}=0
$$

If $n=2$, eq.(4.15) gives $\zeta$ in terms of $\pi_{1}$ and $\pi_{2}$, and the cascade stops here, but if $n>2$ it yields

$$
\pi_{2 n-2}=-\frac{\langle 1 n\rangle}{\mu^{2}} \pi_{1}+\pi_{2} .
$$

The next step is $\dot{\Omega}_{3}=\left\{\Omega_{3}, \mathcal{H}_{T}\right\}_{P B} \equiv \Omega_{4}=0$, which together with (4.14) gives

$$
\psi_{n+2}=\psi_{n-2}+\frac{1}{\mu^{2}}(\langle 1 n\rangle+\langle 2, n-1\rangle) \psi_{n-1}+\frac{1}{\mu^{4}}\langle 1 n\rangle\langle 2 n\rangle \psi_{n}
$$


and, proceeding further, we obtain for the momenta

$$
\begin{aligned}
\dot{\Omega}_{4} & =\left\{\Omega_{4}, \mathcal{H}_{T}\right\}_{P B} \equiv \Omega_{5}=\mu^{4} \pi_{2 n-3}-\langle 1 n\rangle\langle 1, n-1\rangle \pi_{1}+ \\
& +\mu^{2}(\langle 1 n\rangle+\langle 2, n-1\rangle) \pi_{2}-\mu^{4} \pi_{3}\left(1-\delta_{n 3}\right)-2 \sigma \mu^{4} \zeta \delta_{n 3}=0
\end{aligned}
$$

where (4.16) has been taken into account. Again, if $n=3$, the process stops here and we have reproduced the results of section 3. If $n>3$, eq.(4.18) yields

$$
\pi_{2 n-3}=\frac{1}{\mu^{4}}\langle 1 n\rangle\langle 1, n-1\rangle \pi_{1}-\frac{1}{\mu^{2}}(\langle 1 n\rangle\langle 2, n-1\rangle) \pi_{2}+\pi_{3}
$$

and the process goes on.

For illustrative purposes, we complete here the steps that cover the case $n=4$. $\dot{\Omega}_{5}=\left\{\Omega_{5}, \mathcal{H}_{T}\right\}_{P B} \equiv \Omega_{6}=0$, yields

$$
\begin{aligned}
\psi_{n+3} & =\psi_{n-3}+\frac{1}{\mu^{2}}(\langle 3, n-2\rangle+\langle 2, n-1\rangle+\langle 1 n\rangle) \psi_{n-2}+ \\
& +\frac{1}{\mu^{4}}(\langle 2, n-1\rangle\langle 3, n-1\rangle+\langle 1 n\rangle(\langle 2, n-1\rangle+\langle 3 n\rangle)) \psi_{n-1}+ \\
& +\frac{1}{\mu^{6}}\langle 1 n\rangle\langle 2 n\rangle\langle 3 n\rangle \psi_{n},
\end{aligned}
$$

and $\dot{\Omega}_{6}=\left\{\Omega_{6}, \mathcal{H}_{T}\right\}_{P B} \equiv \Omega_{7}=0$ gives $\zeta$ in terms of $\pi_{1}, \pi_{2}, \pi_{3}$, and $\pi_{4}$.

In general, for a fixed $n$, the quadratic dependence of $\mathcal{H}$ on $\pi_{i}$ and $\psi_{i}$, together with the primary constraint $\Omega_{1}$, leads to a set of secondary constraints $\Omega_{k}$ that splits in two classes according to $k$ being even or odd. A constraint $\Omega_{2 j} \quad(j=1, \ldots, n-1)$ is a linear combination of $\psi_{i}$ and gives $\psi_{n+j}$ in terms of $\psi_{n}, \ldots, \psi_{n-j}$. A constraint $\Omega_{2 j-1} \quad(j=2, \ldots, n-1)$ is linear in $\pi_{i}$ and gives $\pi_{2 n-j}$ in terms of $\pi_{1}, \ldots, \pi_{j}$. Finally, $\Omega_{2 n-1}$ fixes the value of $\zeta$ and stops the process.

One can prove that the constraints on the momenta $\Omega_{2 n-1}$ do not contain space derivatives, even though the elements of $\mathcal{M}$ involved in their calculation contain the Laplacian operator. This will be shown in the Appendix A.

Like in (3.26), we take

$$
\psi_{A}=\mathcal{N}_{A \beta} \psi_{\beta}
$$

with indices $\alpha, \beta, \ldots=1, \ldots, n$ and $A, B, \ldots=n+1, \ldots, 2 n-1$. 
Now $\mathcal{N}$ is a $(n-1) \times n$ numerical matrix whose three first rows can be read from (4.14), (4.17) and (4.20). In order to write the elements of $\mathcal{N}$, it is useful to introduce the indices $s=A-n,(s=1, \ldots, n-1)$, that labels the rows of $\mathcal{N}$, and $p=A+\beta-2 n=\beta+s-n,(p=1+s-n, \ldots, s)$, that indicates the odd-diagonals of $\mathcal{N}$. Then, with $P_{p+1}^{s+1} \equiv \mathcal{N}_{n+s, n-s+p}$, one has:

$$
\begin{aligned}
& P_{p+1}^{s+1}=0 \quad \text { for } \quad p<0 \quad ; \quad P_{1}^{s+1}=1 \quad ; \quad P_{2}^{s+1}=\frac{1}{\mu^{2}} \sum_{j_{1}=1}^{s}\left\langle j_{1}, n+1-j_{1}\right\rangle \\
& P_{3}^{s+1}=\frac{1}{\mu^{4}} \sum_{j_{1}=1}^{s-1}\left\langle j_{1}, n+1-j_{1}\right\rangle \sum_{j_{2}=1}^{s-j_{1}}\left\langle j_{1}+j_{2}, n+2-j_{1}-j_{2}\right\rangle \\
& P_{p+1}^{s+1}=\frac{1}{\mu^{2 p}} \sum_{j_{1}=1}^{s-p+1}\left\langle j_{1}, n+1-j_{1}\right\rangle \sum_{j_{2}=1}^{s-p+2-j_{1}}\left\langle j_{1}+j_{2}, n+2-j_{1}-j_{2}\right\rangle \ldots \\
& \ldots \sum_{j_{l}=1}^{s-p+l-j_{1}-\ldots-j_{l-1}}\left\langle j_{1}+\ldots+j_{l}, n+l-j_{1}-\ldots-j_{l}\right\rangle \ldots \\
& \ldots \sum_{j_{p-1}=1}^{s+1-j_{1}-\ldots-j_{p-2}}\left\langle j_{1}+\ldots+j_{p-1}, n+p-1-j_{1}-\ldots-j_{p-1}\right\rangle \\
& \sum_{j_{p}=1}^{s-j_{1}-\ldots-j_{p-1}}\left\langle j_{1}+\ldots+j_{p}, n+p-j_{1}-\ldots-j_{p}\right\rangle \\
& P_{s}^{s+1}=\frac{1}{\mu^{2(s-1)}} \sum_{j_{1}=1}^{2}\left\langle j_{1}, n+1-j_{1}\right\rangle \sum_{j_{2}=1}^{3-j_{1}}\left\langle j_{1}+j_{2}, n+2-j_{1}-j_{2}\right\rangle \ldots \\
& \ldots \sum_{j_{s-2}=1}^{s-1-j_{1}-\ldots-j_{s-3}}\left\langle j_{1}+\ldots+j_{s-2}, n+s-2-j_{1}-\ldots-j_{s-2}\right\rangle \\
& \sum_{j_{s-1}=1}^{s-j_{1}-\ldots-j_{s-2}}\left\langle j_{1}+\ldots+j_{s-1}, n+s-1-j_{1}-\ldots-j_{s-1}\right\rangle \\
& P_{s+1}^{s+1}=\frac{1}{\mu^{2 s}}\langle 1 n\rangle\langle 2 n\rangle \ldots\langle s n\rangle
\end{aligned}
$$

and (4.21) can be rewritten as

$$
\psi_{n+s}=\sum_{p=0}^{s} P_{p+1}^{s+1} \psi_{n-s+p}
$$


The proof of (4.22) and (4.23) is given in Appendix B, were the relation

$$
P_{p+1}^{s+1}=P_{p+1}^{n+p-s} \quad \text { for } \quad p>0
$$

is also proven. Taking $P_{\alpha \beta}=P_{p+1}^{s+1}$, with $\alpha=s+1, \beta=(p+1)-(s+1)+n=p-s+n$, and $P_{1 n}=1, P_{1 \beta}=0$ for $\beta<n$, we can consider $P_{\alpha \beta}$ as a $n \times n$ matrix, that is nothing more than the matrix $\mathcal{N}$ enlarged with a first row, which is symmetric as a consequence of (4.24).

After using the constraints (4.23) to keep only the independent variables, the Lagrangian again is

$$
\mathcal{L}^{2 n}=\dot{\psi}_{\alpha} \overline{\mathcal{K}}_{\alpha \beta} \dot{\psi}_{\beta}+\psi_{\alpha} \overline{\mathcal{M}}_{\alpha \beta} \psi_{\beta}-j \psi_{n}
$$

The $n \times n$ matrices $\overline{\mathcal{K}}$ and $\overline{\mathcal{M}}$ have the same structure in terms of $\mathcal{K}, \mathcal{M}$ and $\mathcal{N}$ given in (3.23) and (3.24). Taking into account (4.22), one checks that

$$
\overline{\mathcal{K}}_{\alpha \beta}=\frac{\mu^{n(n-1)}}{2 M} P_{\beta-n+\alpha}^{\alpha}=\frac{\mu^{n(n-1)}}{2 M} P_{\alpha \beta}=\overline{\mathcal{K}}_{\beta \alpha}
$$

is the generalization of (3.23). For $\overline{\mathcal{M}}$ one has two contributions, with and without the operators $M_{i}^{2}$. The first one can be written, for $\alpha \leq \beta$ as

$$
\overline{\mathcal{M}}_{\alpha \beta}=-\frac{\mu^{n(n-1)}}{2 M} M_{\underline{\beta}}^{2} P_{\alpha \beta},
$$

which displays $M_{\underline{\alpha}}^{2}$ for $\beta \leq \alpha$.

The contribution without $M_{i}^{2}$ is given by

$$
\begin{array}{lll}
\overline{\mathcal{M}}_{\alpha \beta}=0 & \text { if } & \alpha+\beta<n \\
\overline{\mathcal{M}}_{\alpha \beta}=\frac{\mu^{n(n-1)}}{2 M} \mu^{2} P_{\alpha, \beta+1} & \text { if } & \alpha+\beta \geq n ; \alpha, \beta \neq n \\
\overline{\mathcal{M}}_{\alpha \beta}=0 & \text { if } & \alpha \text { or } \beta=n .
\end{array}
$$

The formulas (4.27) and (4.28), are the generalization of (3.24) for arbitrary $n$.

The diagonalization of (4.25) will be accomplished, as in (3.27), by a $n \times n$ real matrix $\mathcal{R}$. We again impose $\mathcal{R}_{n \beta}=1,(\beta=1, \ldots, n)$ to ensure that the current couples to each one of the degrees of freedom. The requirement of simultaneously diagonalizing $\overline{\mathcal{K}}$ and $\overline{\mathcal{M}}$, yields $n(n-1)$ quadratic equations that determine the $n(n-1)$ remaining elements of $\mathcal{R}$. The existence of such a regular $\mathcal{R}$ with real elements is guaranteed by the underlying Lorentz covariance. In fact, the constraints used to get from (4.4) to (4.25) are the (covariant) ones on the fields. In the next section we will see that they can be obtained also from the (covariant) field equations of (4.2). 
They can be implemented on this Lagrangian which can be then diagonalized directly avoiding the decomposition $3+1$. The diagonalizing matrix turns out to be

$$
\begin{aligned}
& \mathcal{R}_{\alpha \beta}=1 \quad ; \quad(\alpha=n) \\
& \mathcal{R}_{\alpha \beta}=(-1)^{n-\alpha} \mu^{-2(n-\alpha)}\langle\beta, \alpha+1\rangle\langle\beta, \alpha+2\rangle \ldots\langle\beta, n\rangle \quad ; \quad(\beta \leq \alpha<n) \\
& \mathcal{R}_{\alpha \beta}=0 \quad ; \quad(\alpha<\beta) \quad,
\end{aligned}
$$

as is shown in the Appendix C. Of course, for $n=3$, this $\mathcal{R}$ is just (3.28).

The diagonalized matrices are now the generalization of (3.29) and (3.30)

$$
\begin{gathered}
\mathcal{R}^{T} \overline{\mathcal{K}} \mathcal{R}=\frac{\mu^{n(n-1)}}{2 M} \operatorname{diag}\left((-1)^{n-1} \frac{\langle 12\rangle\langle 13\rangle \ldots\langle 1 n\rangle}{\mu^{2(n-1)}},(-1)^{n-2} \frac{\langle 12\rangle\langle 23\rangle \ldots\langle 2 n\rangle}{\mu^{2(n-1)}}, \ldots\right. \\
\left.\ldots,(-1)^{n-i} \frac{\langle 12\rangle\langle 13\rangle \ldots\langle 1, i\rangle\langle i, i+1\rangle \ldots\langle i, n\rangle}{\mu^{2(n-1)}}, \ldots, \frac{\langle 1 n\rangle\langle 2 n\rangle \ldots\langle n-1, n\rangle}{\mu^{2(n-1)}}\right),
\end{gathered}
$$

$$
\begin{array}{r}
\mathcal{R}^{T} \overline{\mathcal{M}} \mathcal{R}=\frac{\mu^{n(n-1)}}{2 M} \operatorname{diag}\left((-1)^{n} M_{1}^{2} \frac{\langle 12\rangle \ldots\langle 1 n\rangle}{\mu^{2(n-1)}},(-1)^{n-1} M_{2}^{2} \frac{\langle 12\rangle\langle 23\rangle \ldots\langle 2 n\rangle}{\mu^{2(n-1)}}\right. \\
\left.\ldots,(-1)^{n-i+1} M_{i}^{2} \frac{\langle 12\rangle \ldots\langle 1 i\rangle\langle i, i+1\rangle \ldots\langle i n\rangle}{\mu^{2(n-1)}}, \ldots,-M_{n}^{2} \frac{\langle 1 n\rangle \ldots\langle n-1, n\rangle}{\mu^{2(n-1)}}\right) .
\end{array}
$$

With $\psi_{\alpha}=\mathcal{R}_{\alpha \beta} \phi_{\beta}$, and integrating by parts, the final Lagrangian reads

$$
\begin{aligned}
\mathcal{L}^{2 n} & =(-1)^{n} \frac{1}{2} \frac{1}{\langle 1\rangle} \phi_{1} \llbracket 1 \rrbracket \phi_{1}+(-1)^{n-1} \frac{1}{2} \frac{1}{\langle 2\rangle} \phi_{2} \llbracket 2 \rrbracket \phi_{2}+\ldots \\
& \ldots+(-1)^{n-i+1} \frac{1}{2} \frac{1}{\langle i\rangle} \phi_{i} \llbracket i \rrbracket \phi_{i}+\ldots-\frac{1}{2} \frac{1}{\langle n\rangle} \phi_{n} \llbracket n \rrbracket \phi_{n}-j\left(\phi_{1}+\ldots+\phi_{n}\right),
\end{aligned}
$$

where $\langle i\rangle \equiv \frac{1}{\mu^{n(n-1)}} M \prod_{j \neq i} \frac{1}{\langle i j\rangle \mid}$. This is the result expected from the covariant Ostrogradski method shown in [9]. 


\section{The covariant phase space}

The constraints on the fields and momenta obtained by the Dirac's method define a subset of the phase space in which the solutions to the equations of motion are contained. In non pathological situations, there is a one to one correspondence between points of this subspace and these solutions [12]. In the system under study we have found algebraic constraints on the fields that can be derived also directly from the equations of motion. They define subsets of the configuration space in which the trajectories of the system lie. Now we easily see how these constraints are derived (we put $j=0$ for simplicity).

From (4.4) the following equations of motion are obtained respectively for the fields $\psi_{n+i}, \psi_{i}(1 \leq i<n)$ and $\psi_{n}$.

$$
\begin{aligned}
\mu^{2} \psi_{i} & =\llbracket i+1 \rrbracket \psi_{i+1} \\
\mu^{2} \psi_{n+i} & =\llbracket i \rrbracket \psi_{n+i-1} \\
0 & =\llbracket 1 \rrbracket \psi_{1}+\llbracket n \rrbracket \psi_{2 n-1}
\end{aligned}
$$

From them it is possible to work out the $n-1$ fields $\psi_{i}(n<i<2 n)$ in terms of the $n$ independent fields $\psi_{i}(1 \leq i \leq n)$. This is done by using the first of the equations (5.1) to obtain

$$
\square \psi_{i+1}=\mu^{2} \psi_{i}+m_{i+1}^{2} \psi_{i+1} \quad(1 \leq i<n)
$$

Then iterative use can be made of the second of the equations (5.1), taking (5.2) into account in each step, to obtain the algebraic constraints on the fields (4.14), (4.17) and their general form (4.23). The action (4.25), already depending only on the independent fields, explicitly reads:

$$
\mathcal{L}^{2 n}=\frac{1}{2}\left[-\psi_{n} \llbracket 1 \rrbracket \psi_{1}+\sum_{s=1}^{n-1} \sum_{p=0}^{s} P_{p+1}^{s+1} \psi_{n-s+p}\left(\mu^{2} \psi_{s}-\llbracket s+1 \rrbracket\right) \psi_{s+1}\right]
$$

where the non-diagonal structure is apparent.

The diagonalization matrix (4.29) is suggested by the equations of motion stemming from (5.3) and directly derivable also from (5.1):

$$
\begin{aligned}
\mu^{2(n-1)} \psi_{1} & =\llbracket 2 \rrbracket \cdots \llbracket n \rrbracket \psi_{n} \\
\mu^{2(n-2)} \psi_{2} & =\llbracket 3 \rrbracket \cdots \llbracket n \rrbracket \psi_{n} \\
\cdots \cdots & \quad \cdots \cdots \cdots . . \\
\mu^{2} \psi_{n-1} & =\llbracket n \rrbracket \psi_{n}
\end{aligned}
$$


Defining $n$ new fields $\phi_{a}(1 \leq a \leq n)$, with $\psi_{n}=\sum_{a=1}^{n} \phi_{a}$ and obeying $\llbracket a \rrbracket \phi_{a}=0$, the equations of motion (5.4) become

$$
\begin{aligned}
\mu^{2(n-1)} \psi_{1}= & \langle 2,1\rangle \cdots\langle n, 1\rangle \phi_{1} \\
\mu^{2(n-2)} \psi_{2}= & \langle 3,1\rangle \cdots\langle n, 1\rangle \phi_{1}+\langle 3,2\rangle \cdots\langle n, 2\rangle \phi_{2} \\
\cdots \cdots & \cdots \cdots \cdots \\
\mu^{2} \psi_{n-1}= & \langle n, 1\rangle \phi_{1}+\cdots+\langle n, n-1\rangle \phi_{n-1} \\
\psi_{n}= & \phi_{1}+\cdots+\phi_{n}
\end{aligned}
$$

From these equations it is immediate to read out the elements of the diagonalizing matrix (4.29).

The procedure implemented in this section provides a useful shortcut to get the diagonalized theory. However it is worth noticing that one can not avoid the arduous path followed in section 4 because it guarantees the consistency and the stability of the constraints. Moreover, the Dirac's procedure (when it exists) yields the structure of the reduced phase space (null set of all the constraints, modulo gauge, equipped with the induced non-degenerate symplectic form). The knowledge of the reduced phase space is necessary, for example, when one is interested in using path integral methods because it is on this space where the canonical quantization takes place. Then the covariant method presented in this section complements the results obtained in section 4 besides being specially suitable for the DOF diagonalization. 


\section{Applications to other theories}

The constraint method we have developed for scalar theories can be implemented for HD vector and tensor theories as well. However the canonical $3+1$ procedure described in Section 4 is not convenient for practical applications, mainly when more complex theories as these are considered. The simplified covariant version introduced in Section 5 gets rid of the intermediate constraints $\Omega_{2 j+1}$ on the (non Lorentzcovariant) momenta and obtains the useful ones $\Omega_{2 j}$ on the (covariant) fields by deriving them directly from the (always covariant) equations of motion for all the fields (original HD, auxiliary and multipliers). Moreover when gauge symmetries occur there are further constraints, namely the first class ones, that make more cumbersome the process of working out the Lagrange multipliers. In any case, the covariant phasespace is the method of choice.

\section{HD vectors and differential-forms}

In the case of HD vector theories one can incorporate gauge symmetries to the discussion. A typical example is the generalized QED proposed by F.Bopp and B.Podolsky [13]

$$
\mathcal{L}=-\frac{1}{4} F_{\mu \nu} F^{\mu \nu}-\frac{1}{4 m^{2}} F_{\mu \nu} \square F^{\mu \nu}-j_{\mu} A^{\mu} .
$$

The structure of its constraints, in the gauge-fixed case, has been studied in [3] by a canonical forcefully non-covariant analysis carried out on the Ostrogradski-based order-reduction procedure.

A recasting of a higher-derivative gauge-invariant Yang-Mills theory as a twoderivative one by means of constraints has been done in a non-covariant $3+1$ way [14]. Of course, in the non gauge-fixed theory, second class constraints arise which coexist harmlessly with the first class relevant ones. However we are interested in keeping the explicit Lorentz covariance and, to this end, we make use of the same algebraic manipulations introduced in section 5 .

The theory described by (6.1) can be considered a member of a wider family of theories which share the same general structure of the scalar models (4.1)

$$
\mathcal{L}^{2 n}=-\frac{1}{2} \frac{\mu^{d}}{M} \phi \wedge * \llbracket 1 \rrbracket \llbracket 2 \rrbracket \ldots \llbracket n \rrbracket \phi-j \wedge * \phi \quad,
$$

where $\phi$ is a $s$-form (1-form in the generalized electromagnetism), $\wedge$ is the exterior product, $*$ the Hodge dual (associated to the Minkowski metric), and now the $i \rrbracket$ operators must be understood as combinations of the exterior derivative $d$ and its dual $\delta$ such as

$$
\llbracket i \rrbracket \equiv \delta d+m_{i}^{2} .
$$


Notice that if $m_{n}=0$ the Lagrangian (6.2) describes a gauge theory with gauge transformations $\phi \mapsto \phi+d \Lambda$, where $\Lambda$ is an arbitrary $(s-1)$-form.

With all these considerations, the field equations derived from (6.2) have the same look of equations (5.1) for the scalar model. Moreover, all the arguments that follow this equations in section 5 apply in the same way to the (6.2) HD theory that depends now on differential-form fields. Notice that, despite the gauge invariance of the theory, the order reduction method and the linear redefinition that diagonalize the DOF in the Lagrangian work exactly as in the scalar case.

\section{HD Gravity}

The covariant Ostrogradski order-reduction of the four-derivative gravity leads to a two-derivative equivalent in which the particle DOF can be fully diagonalized in both the Diff-invariant case [8] and with a gauge fixing [15].

The constraint technique for the order-reduction of a pure four-derivative conformally invariant gravitational Lagrangian has been already used in a $3+1$ non-covariant form [16], where further first class constraints from Diff-invariance occur. In a covariant treatment and for the general case including also two-derivative terms [17], a seemingly similar method is adopted where in place of the Lagrange multiplier a less trivial auxiliary field featuring a squared (mass)term is used. A little work shows however that this method is identical to the covariant Ostrogradski's [9]. We illustrate this on the grounds of the scalar model in the Appendix D.

The covariant constraint method introduced in this paper provides a new approach. The most immediate application in higher-derivative gravity regards the linearized theory, usually considered when analyzing the DOF. Take for example the four-derivative Lagrangian

$$
\mathcal{L}=\sqrt{-g}\left[a R+b R^{2}+c R_{\mu \nu} R^{\mu \nu}\right] .
$$

The linearization around the flat Minkowski metric, namely $g_{\mu \nu}=\eta_{\mu \nu}+h_{\mu \nu}$, simplifies it to

$$
\mathcal{L}=\frac{a}{2} h_{\mu \nu} G^{\mu \nu \alpha \beta} r_{\alpha \beta}+r_{\mu \nu} Q^{\mu \nu \alpha \beta}(b, c) r_{\alpha \beta},
$$

where $r_{\mu \nu}$ comes from the linearization of the Ricci tensor

$$
r_{\mu \nu}=\mathcal{R}_{\mu \nu \alpha \beta} h^{\alpha \beta} \equiv \frac{1}{2}\left[h^{\alpha}{ }_{\mu, \nu \alpha}+h^{\alpha}{ }_{\nu, \mu \alpha}-\square h_{\mu \nu}-h_{\alpha, \mu \nu}^{\alpha}\right],
$$

and $G^{\mu \nu \alpha \beta}$ and $Q^{\mu \nu \alpha \beta}$ are numerical matrices:

$$
\begin{aligned}
G^{\mu \nu \alpha \beta} & \equiv \frac{1}{2}\left[\eta^{\mu \nu} \eta^{\alpha \beta}-\eta^{\mu \alpha} \eta^{\nu \beta}-\eta^{\mu \beta} \eta^{\nu \alpha}\right] \\
\mathcal{Q}^{\mu \nu \alpha \beta}(b, c) & \equiv b \eta^{\mu \nu} \eta^{\alpha \beta}+\frac{c}{2}\left[\eta^{\mu \alpha} \eta^{\nu \beta}+\eta^{\mu \beta} \eta^{\nu \alpha}\right] .
\end{aligned}
$$


The field equation for $h_{\mu \nu}$ can be written (omitting indices) in terms of the objects above in the form

$$
\left[G \mathcal{R}+\frac{a}{2} G Q^{-1} G\right] G \mathcal{R} h=0,
$$

Its straightforward to show that the general solution to (6.5) is

$$
h=\mathrm{h}_{1}+\mathrm{h}_{2},
$$

where $\mathrm{h}_{1}$ and $\mathrm{h}_{2}$ satisfy, respectively

$$
G \mathcal{R} \mathrm{h}_{1}=0 \quad ; \quad G \mathcal{R} \mathrm{h}_{2}=-\frac{a}{2} G Q^{-1} G \mathrm{~h}_{2} .
$$

Omitting indices, the order-reduction of the theory by means of a Lagrange multiplier yields the two-derivative local Lagrangian

$$
\mathcal{L}=h_{2}^{\mathrm{t}}\left[\mathcal{R}^{\mathrm{t}} Q G+\frac{a}{2}\right] h_{1}+h_{3}^{\mathrm{t}}\left[\mu^{2} h_{1}-G \mathcal{R} h_{2}\right] .
$$

where $h_{1 \mu \nu}$ is a new field and $h_{3 \mu \nu}$ is the multiplier. Of course, because of the Diff-invariance, first class constraints will remain when the Dirac procedure is carried out. However, it is possible to avoid the use of Dirac method if one looks directly at the space of solutions of the field equations. The field equations for the Lagrangian (6.8) are

$$
\begin{aligned}
& {\left[\mathcal{R}^{\mathrm{t}} Q G+\frac{a}{2}\right] h_{1}-G \mathcal{R} h_{3}=0,} \\
& \mu^{2} h_{3}+\left[\frac{a}{2}+G Q \mathcal{R}\right] h_{2}=0, \\
& \mu^{2} h_{1}-G \mathcal{R} h_{2}=0
\end{aligned}
$$

The last equation leads to $G \mathcal{R} h_{2}=\mu^{2} h_{1}$. Then, the second one expresses algebraically $h_{3}$ in terms of $h_{1}$ and $h_{2}$ :

$$
h_{3}=-\frac{a}{2 \mu^{2}} h_{2}-G Q G h_{1} \text {. }
$$

Using this constraint in the Lagrangian (6.8) we obtain

$$
\mathcal{L}=\frac{a}{2 \mu^{2}} h_{2}^{\mathrm{t}} G \mathcal{R} h_{2}+2 h_{1}^{\mathrm{t}} G Q \mathcal{R} h_{2}-\mu^{2} h_{1}^{\mathrm{t}} G Q G h_{1} .
$$


The last step is again a diagonalization. The hint is given by (6.6)-(6.7), identifying $h=h_{2}$. This leads us to introduce new fields $\mathrm{h}_{1}, \mathrm{~h}_{2}$

$$
\begin{aligned}
& h_{1}=-\frac{a}{2 \mu^{2}} G Q^{-1} G \mathrm{~h}_{2} \\
& h_{2}=\mathrm{h}_{1}+\mathrm{h}_{2},
\end{aligned}
$$

in terms of which the Lagrangian is

$$
\mathcal{L}^{2}=\frac{a}{2 \mu^{2}} \mathrm{~h}_{1} G \mathcal{R} \mathrm{h}_{1}-\frac{a}{2 \mu^{2}} \mathrm{~h}_{2} G \mathcal{R} \mathrm{h}_{2}-\frac{a^{2}}{4 \mu^{2}} \mathrm{~h}_{2} G Q^{-1} G \mathrm{~h}_{2},
$$

where now is clear that $h_{1}$ propagates the massless graviton DOF whereas $h_{2}$ carries a spin 2 with square mass $-a / c$ and a scalar with square mass $a / 2(c+3 b)$.

\section{Conclusions}

We have shown how to deal with $2 n$-derivative relativistic scalar theories by writing them directly as second-order constrained Lagrangians with more fields and suitable Lagrange multipliers. The corresponding canonical conjugate momenta are subject to primary constraints, whose conservation in time gives rise to a finite chain of secondary constraints according to the Dirac's procedure. Though expected, a non trivial result is that these constraints, later used to extract the final DOF, are purely algebraic relations that do not involve the space derivatives.

Once the constraints have been implemented, we are left with a second-order Lagrangian for the DOF of the system. We have performed explicitly the diagonalization for $n=3$, reproducing the result obtained in [9]. Then the procedure has been generalized to arbitrary $n$, namely (4.29). This explicit possibility constitutes a definite advantage over Ostrogradski's method.

The applications to more interesting theories like HD generalized electrodynamics and HD Diff-invariant gravity illustrate also the fact that the order-reducing methods used in the literature fall in two categories: the one based in the covariant Ostrogradski and the one based in the constraints by Lagrange multipliers. The methods based on auxiliary fields with a quadratic term, which may look like a variant of the multipliers, actually belong to the first category and have no obvious manageable extension beyond the four-derivative order.

In vector and tensor field theories where gauge symmetries occur, the corresponding first class constraints live together with the second class ones worked out in this paper and survive the order-reducing procedure as long as gauge fixings are not considered. The method may then prove useful for a detailed analysis of the constraints from gauge (or Diff-)invariance in these HD theories, chiefly of the fate of the scalar and vector constraints of Hamiltonian gravity.

\section{Acknowledgements}

Is a pleasure thanks Dr. F.Barbero for many discussions and comments. 


\section{Appendix A}

We prove, by induction, that the constraints $\Omega_{2 j} \quad(j=1, \ldots, n-1)$ involving the fields, do not contain space derivatives because the Laplacian operators cancel out.

One first sees, by inspection, that this statement is true for $\Omega_{2}$ : the calculation leading to (4.14) is

$$
\Omega_{2} \equiv \sigma\left[\mu^{2} \psi_{n-1}+\left(M_{1}^{2}-M_{n}^{2}\right) \psi_{n}-\mu^{2} \psi_{n+1}\right]=0 \quad,
$$

where the cancellation of the Laplacian operator is apparent, i.e.

$$
M_{1}^{2}-M_{n}^{2}=m_{1}^{2}-m_{n}^{2} \equiv\langle 1 n\rangle
$$

and obviously no summation is understood for repeated indices. Then, let us suppose that, after taking into account the preceding constraints, one has that in the constraint

$\Omega_{2 \alpha}=\sigma\left[\mu^{2 \alpha} \psi_{n-\alpha}+a_{1} \psi_{n-\alpha+1}+a_{2} \psi_{n-\alpha+2}+\ldots+a_{\alpha-1} \psi_{n-1}+a_{\alpha} \psi_{n}-\mu^{2 \alpha} \psi_{n+\alpha}\right]=0$

for $\alpha=1, \ldots, n-2$, the coefficients $a_{1}, \ldots, a_{\alpha}$ are real numbers, as are those found in (4.14), (4.17) and (4.20). We now prove that this is also true for $\Omega_{2 \alpha+1}$. In fact

$$
\begin{aligned}
\Omega_{2 \alpha+1}=\mu^{2 \alpha} \pi_{2 n-\alpha-1}+a_{1} \pi_{2 n-\alpha} & +a_{2} \pi_{2 n-\alpha+1}+\ldots+a_{\alpha-1} \pi_{2 n-2}+a_{\alpha} \pi_{1}- \\
& -\mu^{2} \pi_{\alpha+1}\left(1-\delta_{n, \alpha+1}\right)-2 \sigma \mu^{2} \zeta \delta_{n, \alpha+1}
\end{aligned}
$$

from which

$$
\begin{aligned}
\Omega_{2(\alpha+1)} & \equiv \dot{\Omega}_{2 \alpha+1}=\left\{\Omega_{2 \alpha+1}, \mathcal{H}_{T}\right\}_{P B}= \\
& =\sigma\left[-\mu^{2 \alpha} M_{n-\alpha}^{2} \psi_{n-\alpha}-a_{1} M_{n-\alpha+1}^{2} \psi_{n-\alpha+1}-\ldots-a_{\alpha-1} M_{n-1}^{2} \psi_{n-1}-\right. \\
& -a_{\alpha} M_{n}^{2} \psi_{n}+\mu^{2 \alpha} M_{n+\alpha}^{2} \psi_{n+\alpha}+\mu^{2(\alpha+1)} \psi_{n-\alpha-1}+\mu^{2} a_{1} \psi_{n-\alpha}+\ldots+ \\
& \left.+\mu^{2} a_{\alpha-1} \psi_{n-2}+\mu^{2} a_{\alpha} \psi_{n+1}-\mu^{2(\alpha+1)} \psi_{n+\alpha+1}\right]=0 .
\end{aligned}
$$


The crucial point now is that, when working $\psi_{n+\alpha}$ out of (A.3) and substituting it in (A.5), only differences of squared masses $M_{i}^{2}$ occur as in (A.2), thus cancelling out the operators $\Delta$. Then, by substituting also $\psi_{n+1}$ from (A.1), one gets $\psi_{n+\alpha+1}$ as a sum of linear terms in $\psi_{n}, \psi_{n-1}, \ldots, \psi_{n-\alpha-1}$, the coefficient for the last one being the unity. This ends the inductive proof.

\section{Appendix B}

We proof (4.23) by induction. First note that for $s=1$ it is nothing more than (4.14). Assume then that it holds for $\psi_{n+s}$ with $s \leq n-2$. From the Lagrangian (4.4), one has that the equation of motion for $\psi_{s+1}, s \leq n-2$, is

$$
\mu^{2} \psi_{n+s+1}=\llbracket s+1 \rrbracket \psi_{n+s}=\left(\square+m_{s+1}^{2}\right) \psi_{n+s}
$$

that, with (4.23), gives

$$
\mu^{2} \psi_{n+s+1}=\sum_{p=0}^{s} P_{p+1}^{s+1} \square \psi_{n-s+p}+m_{s+1}^{2} \sum_{p=0}^{s} \psi_{n-s+p}
$$

but the equation of motion for $\psi_{2 n-s+p-1}$ is

$$
\square \psi_{n-s+p}=\mu^{2} \psi_{n-s+p-1}-m_{n-s+p}^{2} \psi_{n-s+p} \quad,
$$

so

$$
\begin{aligned}
\psi_{n+s+1} & =\sum_{p=0}^{s} \frac{\langle s+1, n-s+p\rangle}{\mu^{2}} P_{p+1}^{s+1} \psi_{n-s+p}+\sum_{p=0}^{s} P_{p+1}^{s+1} \psi_{n-s+p-1} \\
& =\frac{\langle s+1, n\rangle}{\mu^{2}} P_{s+1}^{s+1} \psi_{n}+\sum_{p=0}^{s-1}\left[\frac{\langle s+1, n-s+p\rangle}{\mu^{2}} P_{p+1}^{s+1}+P_{p+2}^{s+1}\right] \psi_{n-s+p}+ \\
& +P_{1}^{s+1} \psi_{n-s-1} \\
& =\frac{\langle s+1, n\rangle}{\mu^{2}} P_{s+1}^{s+1} \psi_{n}+\sum_{p=1}^{s}\left[\frac{\langle s+1, n-s+p-1\rangle}{\mu^{2}} P_{p}^{s+1}+P_{p+1}^{s+1}\right] \psi_{n-s+p-1}+ \\
& +P_{1}^{s+1} \psi_{n-s-1},
\end{aligned}
$$


and one gets the desired equation

$$
\psi_{n+s+1}=\sum_{p=0}^{s+1} P_{s+1}^{s+2} \psi_{n-(s+1)+p}
$$

because from (4.22) we have

$$
\begin{aligned}
& P_{1}^{s+1}=P_{1}^{s+2}=1 \quad ; \quad \frac{\langle s+1, n\rangle}{\mu^{2}} P_{s+1}^{s+1}=P_{s+2}^{s+2} \\
& \frac{\langle s+1, n-(s+1)+p\rangle}{\mu^{2}} P_{p}^{s+1}+P_{p+1}^{s+1}=P_{p+1}^{s+2} .
\end{aligned}
$$

The third of this relations is checked by splitting the last term of the sum at the end of $P_{p+1}^{s+2}$. With $j_{p}=s+1-j_{1}-\ldots-j_{p-1}$, it gives the factor $\frac{\langle s+1, n-(s+1)+p\rangle}{\mu^{2}}$ multiplying an expression that can be rewritten as $P_{p}^{s+1}$. The remaining terms in the sum are just $P_{p+1}^{s+1}$.

Now follows the proof of (4.24). Suppose that $\alpha=s+1>n+p-s=r+1=\beta$ and write

$$
\begin{aligned}
P_{p+1}^{s+1} & =\frac{1}{\mu^{2 p}}\left(\sum_{j_{1}=1}^{r-p+1}\left\langle j_{1}, n+1-j_{1}\right\rangle+\sum_{j_{1}=r-p+2}^{s-p+1}\left\langle j_{1}, n+1-j_{1}\right\rangle\right) \times \ldots \\
& \ldots \times\left(\sum_{j_{p-1}=1}^{r-1-j_{1}-\ldots-j_{p-2}}\left\langle j_{1}+\ldots+j_{p-1}, n+p-1-j_{1}-\ldots-j_{p-1}\right\rangle+\right. \\
& \left.+\sum_{j_{p-1}=r-j_{1}-\ldots-j_{p-2}}^{s-1-j_{1}-\ldots-j_{p-2}}\left\langle j_{1}+\ldots+j_{p-1}, n+p-1-j_{1}-\ldots-j_{p}\right\rangle\right) \times \\
& \times\left(\sum_{j_{p}=1}^{r-j_{1}-\ldots-j_{p-1}}\left\langle j_{1}+\ldots+j_{p}, n+p-j_{1}-\ldots-j_{p}\right\rangle+\right. \\
& \left.+\sum_{j_{p}=r+1-j_{1}-\ldots-j_{p-1}}^{s-j_{1}-\ldots-j_{p-2}}\left\langle j_{1}+\ldots+j_{p}, n+p-j_{1}-\ldots-j_{p}\right\rangle\right) .
\end{aligned}
$$


The second term in the last factor is

$$
\langle r+1, s\rangle+\langle r+2, s-1\rangle+\ldots+\langle s-1, r+2\rangle+\langle s, r+1\rangle=0 .
$$

The biggest superior limit for the first term in this last factor, when multiplied by the second term in the previous factor, is determined by the value of $j_{p-1}=$ $r+1-j_{1}-\ldots-j_{p-2}$, so we have that $j_{p}=0$ and this product does not appear because $j_{i}=0 \quad \forall i$. Following this argument, the same happens with all of the second terms, and only remains the product of the first ones that is nothing more than $P_{p+1}^{r+1}$.

\section{Appendix C}

First we will proof (4.30). Taking into account (4.26) and (4.29), we have

$$
\left(\mathcal{R}^{T} \overline{\mathcal{K}} \mathcal{R}\right)_{\alpha \beta}=\frac{\mu^{n(n-1)}}{M} \sum_{\rho \geq \alpha, \sigma \geq \beta} \mathcal{R}_{\rho \alpha} P_{\sigma-n+\rho}^{\rho} \mathcal{R}_{\sigma \beta}
$$

In what follows we omit the overall factor $\frac{\mu^{n(n-1)}}{M}$. Because of $(4.22)$, for $\alpha=\beta=n$ we get

$$
P_{n}^{n}=\frac{\langle 1 n\rangle \ldots\langle n-1, n\rangle}{\mu^{2(n-1)}}
$$

as required.

For $\alpha<\beta=n$, we have to prove that

$$
\begin{aligned}
& P_{n}^{n}+(-1) \frac{\langle\alpha, n\rangle}{\mu^{2}} P_{n-1}^{n-1}+\ldots+(-1)^{n-(\alpha+2)} \frac{\langle\alpha, \alpha+3\rangle \ldots\langle\alpha, n\rangle}{\mu^{2(n-(\alpha+2))}} P_{\alpha+2}^{\alpha+2}+ \\
& +(-1)^{n-(\alpha+1)} \frac{\langle\alpha, \alpha+2\rangle \ldots\langle\alpha, n\rangle}{\mu^{2(n-(\alpha+1))}} P_{\alpha+1}^{\alpha+1}+(-1)^{n-\alpha} \frac{\langle\alpha, \alpha+1\rangle \ldots\langle\alpha, n-1\rangle\langle\alpha, n\rangle}{\mu^{2(n-\alpha)}} P_{\alpha}^{\alpha}=0 .
\end{aligned}
$$

In fact $\frac{\langle\alpha, n\rangle}{\mu^{2}} P_{\alpha}^{\alpha}=P_{\alpha+1}^{\alpha+1}$ because of (B.6), and the two last terms add up to

$$
(-1)^{n-(\alpha+1)} \frac{\langle\alpha, \alpha+2\rangle \ldots\langle\alpha, n-1\rangle}{\mu^{2(n-(\alpha+2))}} \frac{\langle\alpha+1, n\rangle}{\mu^{2}} P_{\alpha+1}^{\alpha+1}
$$


that can be expressed in terms of $P_{\alpha+2}^{\alpha+2}$. Following this procedure step by step we arrive to

$$
P_{n}^{n}-\frac{\langle n-1, n\rangle}{\mu^{2}} P_{n-1}^{n-1}=0
$$

by (B.6).

Thus, the n-th column of the kinetic matrix is null but for the element in the n-th row. Because of the symmetry that gives (4.24), we have for the n-th row

$$
\begin{aligned}
& P_{n}^{n}+(-1) \frac{\langle\alpha, n\rangle}{\mu^{2}} P_{n-1}^{n}+\ldots+(-1)^{n-(\alpha+2)} \frac{\langle\alpha, \alpha+3\rangle \ldots\langle\alpha, n\rangle}{\mu^{2(n-(\alpha+2))}} P_{\alpha+2}^{n}+ \\
& +(-1)^{n-(\alpha+1)} \frac{\langle\alpha, \alpha+2\rangle \ldots\langle\alpha, n\rangle}{\mu^{2(n-(\alpha+1))}} P_{\alpha+1}^{n}+(-1)^{n-\alpha} \frac{\langle\alpha, \alpha+1\rangle \ldots\langle\alpha, n\rangle}{\mu^{2(n-\alpha)}} P_{\alpha}^{n}=0
\end{aligned}
$$

where $\alpha<n$ indicates now the column.

Take now $\beta \leq \alpha<n$. Consider in (C.1) the terms with $\rho=n$, which are (C.6) with $\beta$ in the place of $\alpha$ and give a null contribution. For $\alpha \leq \rho \leq n$ we can write the terms for a given $\rho$ as

$$
\begin{aligned}
& \mathcal{R}_{\rho \alpha}\left[P_{\rho}^{\rho}+P_{\rho-1}^{\rho}(-1) \frac{\langle\beta, n\rangle}{\mu^{2}}+P_{\rho-2}^{\rho}(-1)^{2} \frac{\langle\beta, n-1\rangle\langle\beta, n\rangle}{\mu^{2}}+\ldots\right. \\
& \ldots+P_{\rho-n+(\beta+1)}^{\rho}(-1)^{n-(\beta+1)} \frac{\langle\beta, \beta+2\rangle \ldots\langle\beta, n\rangle}{\mu^{2(n-(\beta+1))}}+ \\
& \left.+P_{\rho-n+\beta}^{\rho}(-1)^{n-\beta} \frac{\langle\beta, \beta+1\rangle \ldots\langle\beta, n\rangle}{\mu^{2(n-\beta)}}\right] .
\end{aligned}
$$

Applying (B.6), (C.7) becomes 


$$
\begin{aligned}
& \mathcal{R}_{\rho \alpha} {\left[\frac{\langle\rho-1, n\rangle}{\mu^{2}} P_{\rho-1}^{\rho-1}+\left\{P_{\rho-1}^{\rho-1}+\frac{\langle\rho-1, n-1\rangle}{\mu^{2}} P_{\rho-2}^{\rho-1}\right\}(-1) \frac{\langle\beta, n\rangle}{\mu^{2}}+\right.} \\
&+\left\{P_{\rho-2}^{\rho-1}+\frac{\langle\rho-1, n-2\rangle}{\mu^{2}} P_{\rho-3}^{\rho-1}\right\}(-1)^{2} \frac{\langle\beta, n-1\rangle\langle\beta, n\rangle}{\mu^{4}}+\ldots \\
& \ldots+\left\{P_{\rho-n+(\beta+1)}^{\rho-1}+\frac{\langle\rho-1, \beta+1\rangle}{\mu^{2}} P_{\rho-n+\beta}^{\rho-1}\right\}(-1)^{n-(\beta+1)} \frac{\langle\beta, \beta+2\rangle \ldots\langle\beta, n\rangle}{\mu^{2(n-(\beta+1))}+} \\
&+\left\{P_{\rho-n+\beta}^{\rho-1}+\frac{\langle\rho-1, \beta\rangle}{\mu^{2}} P_{\rho-n+\beta-1}^{\rho-1}\right\}(-1)^{n-\beta} \frac{\langle\beta, \beta+1\rangle \ldots\langle\beta, n\rangle}{\left.\mu^{2(n-\beta)}\right]=} \\
&=\mathcal{R}_{\rho \alpha} \frac{\langle\rho-1, \beta\rangle}{\mu^{2}}\left[P_{\rho-1}^{\rho-1}+P_{\rho-2}^{\rho-1}(-1) \frac{\langle\beta, n\rangle}{\mu^{2}}+P_{\rho-3}^{\rho-1}(-1)^{2} \frac{\langle\beta, n-1\rangle\langle\beta, n\rangle}{\mu^{4}}+\ldots\right. \\
& \ldots+P_{\rho-n+\beta}^{\rho-1}(-1)^{n-(\beta+1)} \frac{\langle\beta, \beta+2\rangle \ldots\langle\beta, n\rangle}{\mu^{2(n-(\beta+1))}+} \\
&\left.\quad+P_{\rho-n+\beta-1}^{\rho-1}(-1)^{n-\beta} \frac{\langle\beta, \beta+1\rangle \ldots\langle\beta, n\rangle}{\mu^{2(n-\beta)}}\right]
\end{aligned}
$$

The expression inside the brackets is the contribution to (C.1) from the terms with $\rho-1$ divided by $\mathcal{R}_{\rho-1, \alpha}$. So, if the contribution for $\rho$ is zero, it is zero too for $\rho-1$ as long as $\langle\rho-1, \beta\rangle \neq 0$. When $\beta<\alpha$, we have seen that for $\rho=n$ we have a null contribution and then it is null for $\rho=n-1$, and so is down to $\rho=\alpha+1$ and $\rho=\alpha$. All the contributions being zero, the kinetic matrix is diagonal because of the symmetry. For $\beta=\alpha$ the same procedure works well until we reach $\rho=\alpha+2$, so that the contribution for $\rho=\alpha+1$ is zero, but here the chain stops because $\langle\rho-1, \beta\rangle=0$ in the next step, and we get

$$
\begin{aligned}
\left(\mathcal{R}^{T} \overline{\mathcal{K}} \mathcal{R}\right)_{\alpha \alpha} & =\frac{\mu^{n(n-1)}}{M} \sum_{\rho \geq \alpha} \mathcal{R}_{\alpha \alpha} P_{\sigma-n+\alpha}^{\alpha} \mathcal{R}_{\sigma \alpha}= \\
& =\frac{\mu^{n(n-1)}}{M}(-1)^{n-\alpha} \frac{\langle\alpha, \alpha+1\rangle \ldots\langle\alpha, n\rangle}{\mu^{2(n-\alpha)}}\left[P_{\alpha}^{\alpha}+(-1) \frac{\langle\alpha, n\rangle}{\mu^{2}} P_{\alpha-1}^{\alpha}+\right. \\
& +(-1)^{2} \frac{\langle\alpha, n-1\rangle\langle\alpha, n\rangle}{\mu^{4}} P_{\alpha-2}^{\alpha}+\ldots \\
& \ldots+(-1)^{n-\alpha+1} \frac{\langle\alpha, \alpha+2\rangle \ldots\langle\alpha, n\rangle}{\mu^{2(n-(\alpha+1))}} P_{\alpha-n+\alpha+1}^{\alpha}+ \\
& \left.+(-1)^{n-\alpha} \frac{\langle\alpha, \alpha+1\rangle \ldots\langle\alpha, n\rangle}{\mu^{2(n-\alpha)}} P_{\alpha-n+\alpha}^{\alpha}\right] .
\end{aligned}
$$


To get (4.30), we only need to prove that the square bracket in (C.9) gives

$$
\frac{\langle 1, \alpha\rangle \ldots\langle\alpha-1, \alpha\rangle}{\mu^{2(\alpha-1)}}
$$

To obtain this result, we apply to (C.9) the same procedure used in (C.8) and get

$$
\frac{\langle\alpha-1, \alpha\rangle}{\mu^{2}}\left[P_{\alpha-1}^{\alpha-1}+(-1) \frac{\langle\alpha, n\rangle}{\mu^{2}} P_{\alpha-2}^{\alpha-1}+\ldots+(-1)^{n-\alpha} \frac{\langle\alpha, \alpha+1\rangle \ldots\langle\alpha, n\rangle}{\mu^{2(n-\alpha)}} P_{\alpha-n+\alpha-1}^{\alpha-1}\right]
$$

We observe that $\alpha-n+\alpha \leq \alpha$. If $\alpha-n+\alpha<0$ some of the terms in (C.9) are zero. If $\alpha-n+\alpha=l \geq 0$ we can repeat for (C.11) the same operation we did on (C.9), and, after $l+1$ steps, the last term is zero. After the step $\alpha-2$ we finally get

$$
\frac{\langle 2, \alpha\rangle \ldots\langle\alpha-1, \alpha\rangle}{\mu^{2(\alpha-2)}}\left[P_{2}^{2}+(-1) \frac{\langle\alpha, n\rangle}{\mu^{2}} P_{1}^{2}\right]=\frac{\langle 1, \alpha\rangle \ldots\langle\alpha-1, \alpha\rangle}{\mu^{2(\alpha-1)}}
$$

as required.

Now we show how to get (4.31). We will study the situation when $\beta \leq \alpha$ as long as the mass matrix is symmetric, and again omit $\frac{\mu^{n(n-1)}}{M}$, so we consider

$$
\sum_{\rho \geq \alpha, \sigma \geq \beta} \mathcal{R}_{\rho \alpha} \overline{\mathcal{M}}_{\rho \sigma} \mathcal{R}_{\sigma \beta}
$$

Take $\alpha=n$. Then, if $\beta=n$, one gets

$$
-M_{n}^{2}\langle 1, n\rangle\langle 2, n\rangle \ldots\langle n-1, n\rangle
$$

for (C.13). If $\beta<n$ it is the same expression obtained for the kinetic matrix with the factor $-M_{n}^{2}$, so it vanishes.

For $\alpha=n-1$ we have in (C.13) $\rho=n, n-1$. The first value gives the same result as in the kinetic case but with the factor $-M_{n}^{2}$, and it vanishes again for $\beta \leq \alpha$. For $\rho=n-1$ we have contributions with and without $\mu^{2}$ : 


$$
\begin{aligned}
& \mu^{2}\left[P_{n-1}^{n}(-1) \frac{\langle\beta, n\rangle}{\mu^{2}}+P_{n-2}^{n}(-1)^{2} \frac{\langle\beta, n-1\rangle\langle\beta, n\rangle}{\mu^{4}}+\ldots\right. \\
& \left.\ldots+P_{\beta}^{n}(-1)^{n-\beta} \frac{\langle\beta, \beta+1\rangle \ldots\langle\beta, n\rangle}{\mu^{2(n-\beta)}}\right]+\left(-M_{n}^{2}\right) P_{n-1}^{n-1}+ \\
& +\left(-M_{n-1}^{2}\right)\left[P_{n-2}^{n-1}(-1) \frac{\langle\beta, n\rangle}{\mu^{2}}+P_{n-3}^{n-1}(-1)^{2} \frac{\langle\beta, n-1\rangle\langle\beta, n\rangle}{\mu^{4}}+\ldots\right. \\
& \left.\ldots+P_{\beta-1}^{n-1}(-1)^{n-\beta} \frac{\langle\beta, \beta+1\rangle \ldots\langle\beta, n\rangle}{\mu^{2(n-\beta)}}\right] .
\end{aligned}
$$

Inside the bracket for $\mu^{2}$, we have the same expression obtained for the kinetic part when $\rho=n$ but without the first term, so it gives $-P_{n}^{n} \mu^{2}$. Adding it to the next, we have

$$
-P_{n}^{n} \mu^{2}+P_{n-1}^{n-1}\left(-M_{n}^{2}\right)=-\frac{\langle n-1, n\rangle}{\mu^{2}} P_{n-1}^{n-1} \mu^{2}+P_{n-1}^{n-1}\left(-M_{n}^{2}\right)=P_{n-1}^{n-1}\left(-M_{n-1}^{2}\right)
$$

that, together with the rest of (C.15), is the same occurring in the kinetic case aside the factor $-M_{n-1}^{2}$. So it is zero if $\beta<\alpha$, and for $\beta=\alpha=n-1$ one has the result needed in (4.31).

Similar rearrangements are used for the rest of the matrix. For example, for $\alpha=n-2$ one has two relations

$$
\begin{aligned}
& -P_{n-1}^{n-1} \mu^{2}+P_{n-2}^{n-2}\left(-M_{n}^{2}\right)=P_{n-2}^{n-2}\left(-M_{n-2}^{2}\right) \\
& -P_{n-2}^{n-1} \mu^{2}+P_{n-2}^{n-2} \mu^{2}+P_{n-3}^{n-2}\left(-M_{n-1}^{2}\right)= \\
& =-\frac{\langle n-2, n-1\rangle}{\mu^{2}} P_{n-3}^{n-2} \mu^{2}+P_{n-3}^{n-2}\left(-M_{n-1}^{2}\right)=P_{n-3}^{n-2}\left(-M_{n-2}^{2}\right),
\end{aligned}
$$

and one arrives again at the kinetic result with the factor $-M_{n-2}^{2}$.

\section{Appendix D}

Consider the four-derivative Lagrangian,

$$
\mathcal{L}^{4}=-\frac{1}{2} \frac{1}{\langle 12\rangle} \phi \llbracket 1 \rrbracket \llbracket 2 \rrbracket \phi-j \phi
$$


which also reads

$$
\mathcal{L}^{4}=-\frac{1}{2} \frac{1}{\langle 12\rangle}\left[p \phi^{2}+s \phi(\square \phi)+(\square \phi)^{2}\right]-j \phi \quad,
$$

where $p=m_{1}^{2} m_{2}^{2}$ and $s=m_{1}^{2}+m_{2}^{2}$. The covariant Ostrogradski method, in a slightly less refined version that the one presented in [9], would define a conjugate generalized momentum $\pi=\frac{\partial \mathcal{L}}{\partial(\square \phi)}$. The Legendre transformation performed on it leads to a Hamiltonian-like density from which the following two-derivative Helmholtz Lagrangian is derived

$$
\mathcal{L}_{H}=\pi \square \phi+\frac{1}{2}\langle 12\rangle \pi^{2}+\frac{1}{2}\langle 12\rangle \phi^{2}+\frac{1}{2} s \pi \phi
$$

On the other hand, by using the auxiliary field technique of [17] the higher-derivative term is brought to second order by writing (D.2) as

$$
\mathcal{L}^{4}=-\frac{1}{2} \frac{1}{\langle 12\rangle}\left[p \phi^{2}+s \phi(\square \phi)+\Lambda(\square \phi)-\frac{1}{4} \Lambda^{2}\right]-j \phi \quad,
$$

where the equation of motion for $\Lambda$ recovers (D.2) when substituted back in (D.4). Now, in spite of their quite different look, (D.3) and (D.4) are related by the simple field redefinition $\pi=-\frac{1}{2} \frac{1}{\langle 12\rangle}(\Lambda+s \phi)$. 


\section{REFERENCES}

[1] K.Jansen, J.Kuti and Ch.Liu, Phys.Lett.B309(1993)119;

Phys.Lett.B309(1993)127.

[2] B.Podolski and P.Schwed, Rev.Mod.Phys.20(1948)40.

K.S.Stelle, Gen.Rel.Grav.9(1978)353.

[3] A.Bartoli and J.Julve, Nucl.Phys.B425(1994)277.

[4] K.S.Stelle, Phys.Rev.D16(1977)953.

I.L.Buchbinder, S.D.Odintsov and I.L.Shapiro, Effective Action in Quantum Gravity,

[5] D.J.Gross and E.Witten, Nucl.Phys.B277(1986)1.

R.R.Metsaev and A.A.Tseytlin, Phys.Lett.B185(1987)52 .

M.C.Bento and O.Bertolami, Phys.Lett.B228(1989)348.

[6] N.D.Birrell and P.C.W.Davies, Quantum Fields in Curved Space,

Cambridge Univ.Press(1982). (IOP, Bristol and Philadelphia, 1992).

[7] M.Ferraris and J.Kijowski, Gen.Rel.Grav. 14(1982)165.

A.Jakubiec and J.Kijowski, Phys.Rev.D37(1988)1406.

G.Magnano, M.Ferraris and M.Francaviglia, Gen.Rel.Grav.19(1987)465;

J.Math.Phys.31(1990)378; Class.Quantum.Grav.7(1990)557.

[8] J.C.Alonso, F.Barbero, J.Julve and A.Tiemblo,

Class.Quantum Grav.11(1994)865.

[9] F.J.de Urries and J.Julve, J.Phys.A: Math.Gen.31(1998)6949.

[10] T.Nakamura and S.Hamamoto, Prog.Theor.Phys.95(1996)469.

[11] P.A.M.Dirac, Canad.J.Math.2(1950)129; Proc.Roy.Soc.(London)A246(1958)326.

[12] E.Witten and Č.Crnković, Covariant description of canonical formalism in geometrical theories in Three Hundred Years of Gravitation S.W.Hawking and W.Israel Eds. Cambridge University Press (1989) 676-684.

J.Fernando Barbero G. and Eduardo J.S. Villaseñor, Nucl.Phys.B600(2001)423 and references therein.

[13] F.Bopp, Ann.Physik,38(1940)345.

B.Podolsky, Phys.Rev.62(1942)68.

B.Podolsky and C.Kikuchi, Phys.Rev.65(1944)228.

B.Podolsky and P.Schwed, Rev.Mod.Phys.20(1948) 40.

[14] M.Kaku, Phys.Rev.D27(1983)2819.

[15] A.Bartoli, J.Julve and E.J.Sánchez, Class.Quantum Grav.16(1999)2283.

[16] M.Kaku, Nucl.Phys.B203(1982)285.

[17] A.Hindawi, B.A.Ovrut and D.Waldram, Phys.Rev.D53(1996)5583. 